\title{
9. MIDDLE CRETACEOUS CALCAREOUS NANNOFOSSILS FROM THE WESTERN PACIFIC (LEG 129): EVIDENCE FOR PALEOEQUATORIAL CROSSINGS ${ }^{1}$
}

\author{
Elisabetta $\mathrm{Erba}^{2}$
}

\begin{abstract}
Middle Cretaceous calcareous nannofossils were quantitatively studied at Sites 800,801 , and 802 of Leg 129 . Samples were selected after careful inspection of the total abundance and preservation of nannofloras in pelagic sediments in order to analyze only the best-preserved assemblages and exclude major secondary modifications of the nannofloras due to dissolution/diagenesis. Nannofossil data were compared with radiolarian distribution and paleolatitude values to trace the response of planktonic communities to plate motions toward the paleoequator.

Aptian to Cenomanian calcareous nannofloras record changes in composition, with sharp increases in abundance of Biscutum constans and Zygodiscus erectus. Both species were previously interpreted as high-fertility indicator characteristic of the paleoequatorial belt of the Pacific basin and of upwelling sites.

At Sites 800 and 801 the increases in abundance of these indices correspond to increases of radiolarians. At both sites this change was recorded when paleolatitude values pass from $10^{\circ} \mathrm{S}$ to $5^{\circ} \mathrm{S}$, and therefore seems to mark the southern edge of the paleoequatorial divergence. At paleolatitudes of approximately $2^{\circ} \mathrm{S}$, in the core of the upwelling zone, calcareous nannofossils disappeared and were replaced by extremely abundant radiolarians.

Site 800 reached the high-fertility belt during the late middle Albian and the core of the paleoequatorial divergence in the Cenomanian. Site 801 approached the upwelling belt during the late Albian and reached the inner part of the divergence in the Cenomanian.

Data from Site 802 are less clear; here, calcareous nannofossils are abundant but poorly preserved in the upper Aptian-Cenomanian interval. The high-fertility indices do not show increases in abundance and indeed, paleolatitude values point to a location south of the paleoequatorial upwelling zone. However, nannofossil assemblages might be partially altered by dissolution because of the deeper paleoenvironment.
\end{abstract}

\section{INTRODUCTION}

The Mesozoic sediments drilled at Sites 800, 801, and 802 of Leg 129 (Fig. 1) contain calcareous nannofossils displaying wide fluctuations in abundance, preservation, and species diversity (see Erba and Covington, this volume). The three sites were drilled at great water depths (Table 1) and oceanic sequences were recovered consisting mainly of chert, porcellanite, radiolarite, and claystone with a major influence by volcaniclastic turbidites. In such a scenario radiolarians are the most abundant group, and nannofossil distribution is restricted to calcareous intervals. At the three sites, pelagic limestone and chalk are limited to the middle Cretaceous interval, where nannofossils are better preserved and overwhelm radiolarians in abundance. They are absent in the Berriasian-Barremian interval, whereas the Upper Cretaceous interval is barren of nannofossils at Sites 800 and 801 but contains rare to abundant assemblages at Site 802 (Fig. 2).

Quantitative analyses of nannofloras were performed on the better preserved samples of the Aptian-Cenomanian interval from Sites 800 , 801 , and 802 to point out possible fluctuations of assemblage composition reflecting paleoenvironmental changes. A similar study was carried out by Roth (1981) at Deep Sea Drilling Project (DSDP) Sites $463,464,465$, and 466 , where he identified a high-fertility nannofossil assemblage in the equatorial belt of the Central Pacific. In the present study nannofossil abundances are used in an attempt to recognize such an assemblage and the time of the equatorial crossings experienced by Sites 800,801 , and 802 . Moreover, nannofossil information will be combined with the abundance changes of radiolarians in order to test the potential of using planktonic microfossils to trace plate motions with respect to the paleoequator. These paleon-

'Larson, R. L., Lancelot, Y., et al., 1992. Proc. ODP, Sci. Results, 129: College Station, TX (Ocean Drilling Program).

${ }^{2}$ Dipartimento di Scienze della Terra, Via Mangiagalli 34, I-20133 Milano, Italy. tological data might be used as independent evidence of the motion of the Pacific plate during the Mesozoic.

\section{CRETACEOUS NANNOFOSSIL PALEOCEANOGRAPHY}

In the past decade calcareous nannofossils have increasingly been used as tracers of Mesozoic oceans because they provide an excellent proxy record of chemical and physical changes of surface water masses. Roth $(1986,1989)$ presented compilations of data collected for the temporal and spatial distribution of Jurassic and Cretaceous nannofloras and outlined the major steps in their diversification and evolution. The most striking feature in the Mesozoic nannofossil history is their growing importance as producers of pelagic carbonates. The oldest consistent occurrence of calcareous nannofossils is dated as Carnian, and Late Triassic nannofloras have been reported from different basins (e.g., Bown, 1987; Bralower et al., 1991). These assemblages were poorly diversified and species diversity progressively increased through the Jurassic (Roth, 1986, 1989; Erba, Castradori, and Cobianchi, in press). A dramatic increase in abundance and diversity of calcareous nannofloras in the Upper Jurassic is responsible for the shift of carbonate deposition from shallow sites to open oceans. This event is most evident in the Tithonian of the Tethyan area, where a superbloom of nannoconids marked the lithologic change from dominantly or partially siliceous sequences of Middle and Late Jurassic age to widespread highly calcareous sediments in the Lower Cretaceous.

Detailed investigations of nannofloras have been carried out mainly in middle Cretaceous sequences, whereas the Lower and the Upper Cretaceous need more information. Quantitative analyses of Aptian-Cenomanian calcareous nannofossils were performed by Roth and coworkers (Roth, 1981, 1986, 1989; Roth and Bowdler, 1981; Roth and Krumbach, 1986; Thierstein and Roth, 1991) on several sequences drilled in the Atlantic, Indian, and Pacific oceans and land sections in England, France, and Texas. Coeval nannofloras 


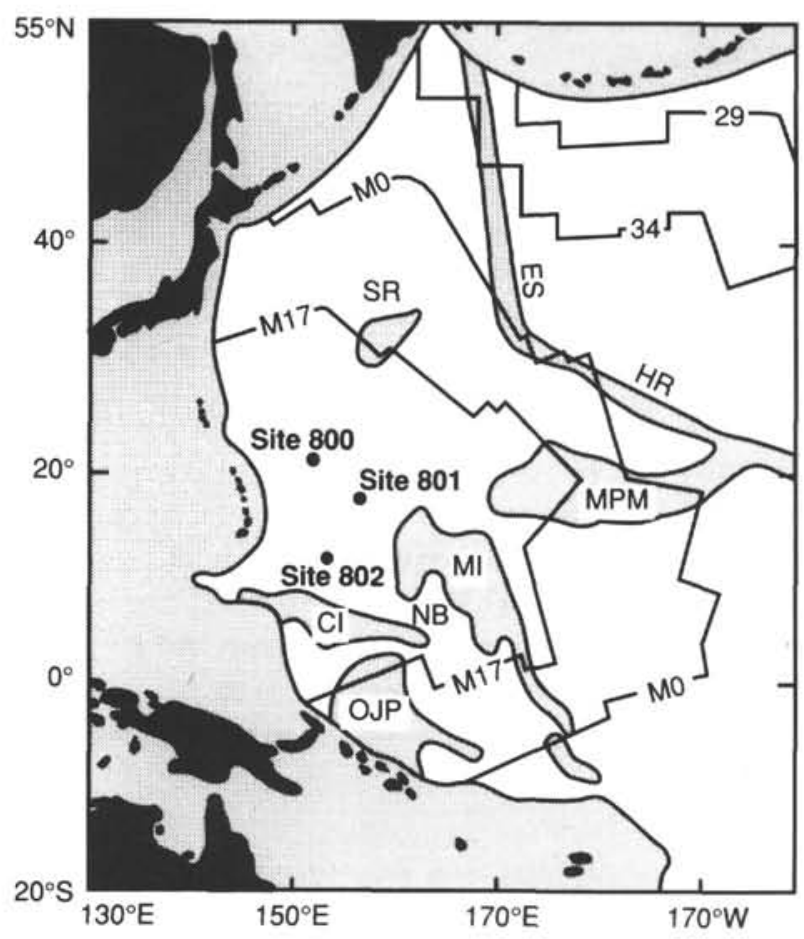

Figure 1. Location of Sites 800,801 , and 802 drilled during Leg 129. Bedrock isochrons are determined from magnetic anomaly lineation mapping of the Pacific plate. Unshaded areas represent normal Pacific oceanic crust, shaded areas represent volcanic edifices with thickened crustal sections, as well as younger areas beyond the Pacific subduction zones (after Lancelot, Larson, et al., 1990). $S R=$ Shatsky Rise; ES = Emperor Seamounts; HR = Hawaiian Ridge; $\mathrm{MPM}=$ Mid-Pacific Mountains; $\mathrm{MI}=$ Marshall Islands; $\mathrm{Cl}=$ Caroline Islands; NB = Nauru Basin; OJP = Ontong Java Plateau.

from marginal seas were quantitatively studied by Watkins (1986, $1989)$ in the Western Interior, by Mutterlose $(1987,1989,1991)$ in Northern Germany, by Premoli Silva et al. (1989) and Erba (1986, 1987, in press) in central Italy, and by Erba et al. (1989) and Erba, Castradori, Guasti, and Ripepe (in press) in Southern England.

These studies have contributed to better understanding the nannofossil biogeography and correlation of these data with sedimentology, organic and inorganic geochemistry, and distribution of other fossil groups allowed interpretations of changes in nannofloral composition related to primary paleoenvironmental signals. Interpretations converge on middle Cretaceous nannofloras being controlled mainly by relative changes in surface water fertility. Paleolatitudinal gradients only partially controlled the distribution of nannofossil assemblages but a few temperature-controlled nannofossil indices were tentatively identified. A more developed diversification was noted in oceanic than in marginal assemblages.

Particular attention to diagenesis was paid by Thierstein (1980, 1981), Roth (1984), and Thierstein and Roth (1991), who outlined the importance of preservation estimates to avoid misleading results caused by diagenesis. In fact, nannofossil preservation is a measure of carbonate dissolution and recrystallization and can be used to reconstruct the diagenetic history of pelagic carbonates. Various Cretaceous nannofossil taxa have been ranked with respect to resistance to dissolution/diagenesis (Thierstein, 1980, 1981; Roth, 1984; Roth and Krumbach, 1986). Close inspection of taxonomic composition and preservation is needed to discern between primary differences and secondary changes in nannofossil assemblages.

As regards the Pacific Ocean, Roth (1981) studied the nannofossil assemblages recorded at Sites $463,464,465$, and 466 in the middle Cretaceous interval. Nannofloras displayed changes in composition
Table 1. Location and number of samples selected for counts of calcareous nannofossils in middle Cretaceous sediments at

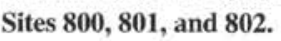

\begin{tabular}{lccc}
\hline & Site 800 & 801 & 802 \\
\hline Latitude (N) & $21^{\circ} 55.38^{\prime}$ & $18^{\circ} 38.568^{\prime}$ & $12^{\circ} 5.778^{\prime}$ \\
Longitude (E) & $152^{\circ} 19.37^{\prime}$ & $156^{\circ} 21.57^{\prime}$ & $153^{\circ} 12.6258^{\prime}$ \\
Water depth (mbsf) & 5686.0 & 5673.8 & 5968.6 \\
$\begin{array}{l}\text { Initially prepared } \\
\text { samples }\end{array}$ & 193 & 80 & 27 \\
$\begin{array}{l}\text { Samples selected for } \\
\text { analysis }\end{array}$ & 20 & 12 & 6 \\
\hline
\end{tabular}

and major increases in abundance of Biscutum constans, Zygodiscus erectus, and Zygodiscus spp. were depicted. These taxa became particularly abundant when sites were in the equatorial divergence area and consequently were interpreted as indices of high fertility in the equatorial upwelling belt. In particular, the nannofossil assemblages at Site 463 showed sharp increases in abundance of $B$. constans and $Z$. erectus when the site was moving toward the paleoequator. The quantitative data published by Roth (1981) are replotted in Figure 3 along with the lithology, the nannofossil biostratigraphy, and the paleolatitude changes inferred from the model of Lancelot and Larson (1975) and Lancelot (1978) (Roth, 1981; Thiede et al., 1981). It must be noted that $B$. constans increases in abundance and reaches the maximum value before $Z$. erectus.

The affinity of both $B$. constans and $Z$. erectus for high-fertility conditions were later confirmed in a number of studies carried out independently in different areas (Erba, 1986, 1987, in press; Roth and Krumbach, 1986; Watkins, 1989; Premoli Silva et al., 1989; Erba et al., 1989; Erba, Castradori, Guasti, and Ripepe, in press; Roth, 1989) (Fig. 4).

\section{MATERIALS AND METHODS}

Nannofossils counts were carried out using a polarizing light microscope. Smear slide preparation was kept simple to retain the original sediment composition. Centrifuge concentration and ultrasonic cleaning may selectively alter the nannofossil composition and modify the mineralogical composition of the sediments as well.

An accurate selection of the best preserved intervals was carried out through the middle Cretaceous recovered at Sites 800,801 , and 802 , and samples affected by strong dissolution and/or overgrowth were not considered for quantitative analyses. Only 38 samples out of 300 were found to be suitable for this study, as shown in Table 1. Particular attention was paid to the volcaniclastic units recovered at the three sites in order to detect the sporadic pelagic carbonate layers interbedded in the turbidites.

In each of the selected samples, counts of 300 nannofossil specimens were performed on randomly chosen fields of view, at $1250 \times$ magnification. The percentages of the most abundant species at the three sites are listed in Table 2.

\section{RESULTS}

Calcareous nannofossil assemblages show some major changes through time in the selected samples from Sites 800,801 , and 802 (Table 2). The total abundance of nannofloras does not vary significantly because only the layers with the most abundant nannofossils were selected from pelagic calcareous intervals for quantitative analyses. For the same reason preservation is always moderate to good, even in the volcaniclastic turbidite units, because only nicely preserved samples were analyzed. Good preservation is indicated also 


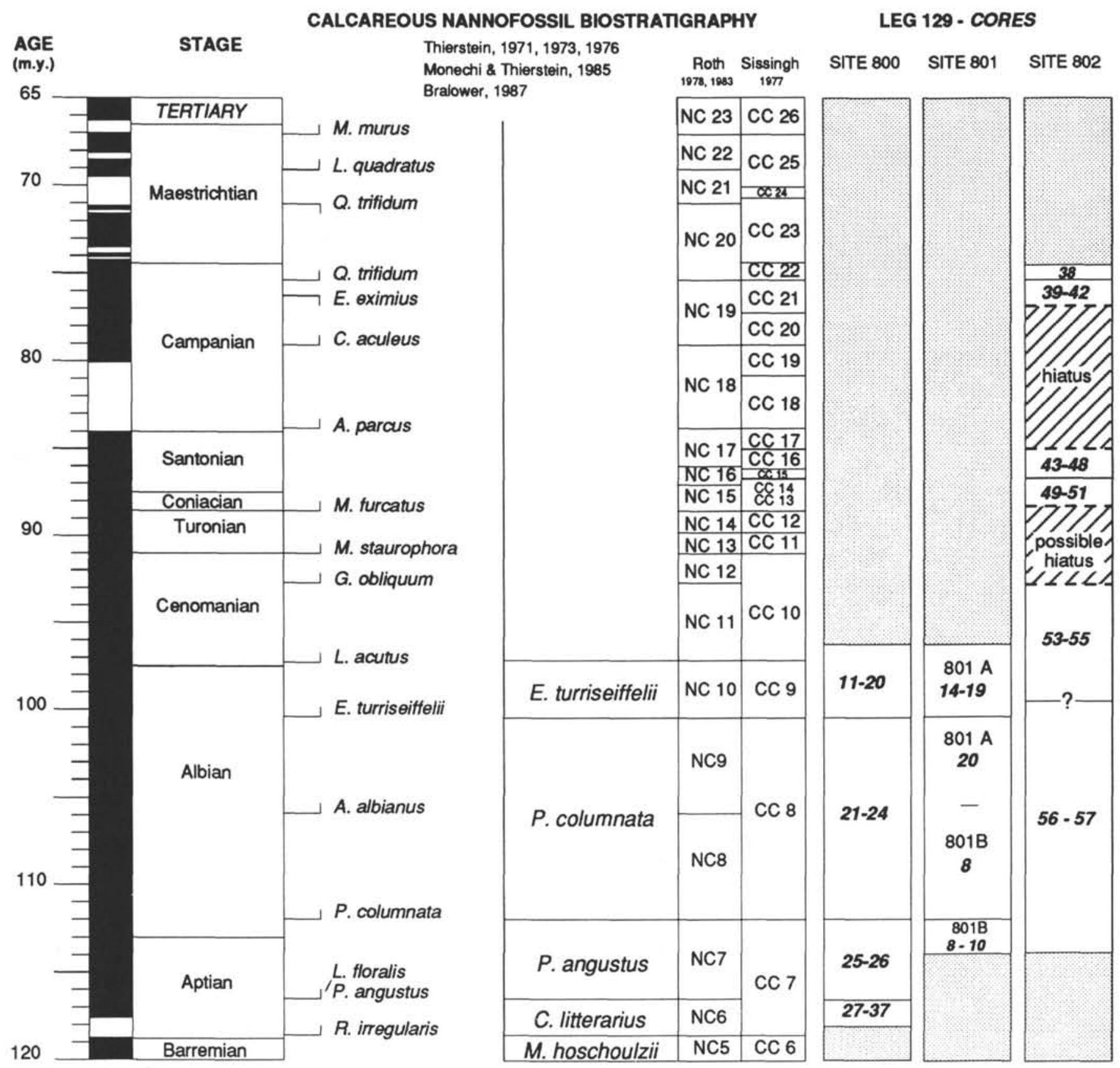

Figure 2. Synthesis of nannofossil biostratigraphy applied to the Cretaceous sediments recovered at Sites 800, 801, and 802 (Erba and Covington, this volume). Absolute ages, chronology, and magnetic polarity sequence after Kent and Gradstein (1985).

by the low abundance of the dissolution- and diagenesis-resistant species Watznaueria barnesae and of other robust taxa as well. A percentage of $40 \% \mathrm{~W}$. barnesae was proposed as boundary value to recognize assemblages altered by secondary modifications and therefore not suitable for paleoceanographic studies (Thierstein, 1981; Roth and Bowdler, 1981; Roth, 1984; Roth and Krumbach, 1986). However, Roth and Krumbach (1986), Erba et al. (1989), Erba, Castradori, Guasti, and Ripepe (in press), and Roth (1989) noted that high-fertility assemblages are significantly impoverished in W. barnesae. As shown in Table 2,W. barnesae occurs with low percentages in samples from Sites 800 and 801 , but this taxon is always more abundant than $40 \%$ in samples from Site 802 .

The nannofossil fertility indices Biscutum constans, Zygodiscus erectus, and Zygodiscus spp. were recognized in all the selected samples, and their distribution records dramatic changes through time. Major increases in abundance of these taxa were identified at Sites 800 and 801 , but not at Site 802 . Other nannofossil taxa displaying fluctuations in abundance are Cretarhabdus spp., Rucinolithus irregularis, and Discorhabdus rotatorius.

The composition of calcareous nannofloras will be discussed site by site in stratigraphic order, from the oldest to the youngest samples.

\section{Site $\mathbf{8 0 0}$}

At Site 800 calcareous nannofossils dominate the pelagic sedimentation from the early Aptian through the Cenomanian. Figure 5 shows the fluctuations in abundance of the most abundant taxa recognized in the middle Cretaceous at this site (Table 2). 
Table 2. Percentage of the most abundant nannofossil taxa in middle Cretaceous sediments recovered at Sites 800, 801, and 802.

\begin{tabular}{|c|c|c|c|c|c|c|c|c|c|c|c|c|c|c|c|c|c|c|c|c|c|c|c|c|c|c|c|c|c|c|}
\hline Age & $\begin{array}{c}\text { Nannofossil } \\
\text { biozone }\end{array}$ & $\begin{array}{l}\text { Core, section, } \\
\text { interval (cm) }\end{array}$ & 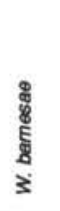 & 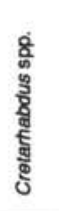 & 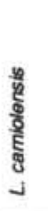 & $\begin{array}{l}\text { है } \\
\text { ूू } \\
8 \\
\infty \\
\infty\end{array}$ & 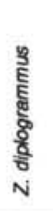 & $\begin{array}{l}\frac{\mathrm{g}}{\mathrm{g}} \\
\frac{8}{6} \\
\mathrm{~N}\end{array}$ & 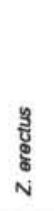 & 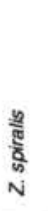 & 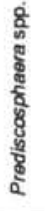 & $\begin{array}{l}\frac{3}{\delta} \\
\frac{0}{0} \\
\frac{0}{0} \\
0\end{array}$ & 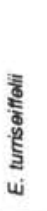 & 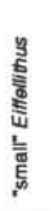 & 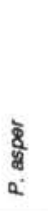 & 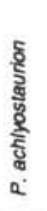 & 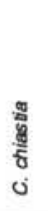 & 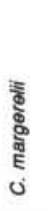 & 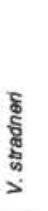 & 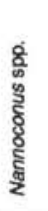 & 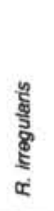 & 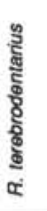 & 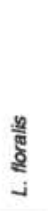 & 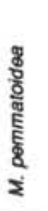 & 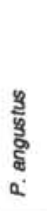 & 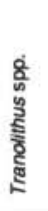 & 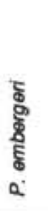 & 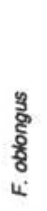 & 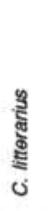 & $\begin{array}{l}\text { कूँ. } \\
\text { है } \\
\text { हूँ } \\
\text { के } \\
\text { نे }\end{array}$ \\
\hline \multirow{8}{*}{ 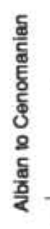 } & \multicolumn{2}{|r|}{$120-800 \mathrm{~A} \cdot 11 \mathrm{R}-1,30$} & $\begin{array}{l}40.0 \\
420\end{array}$ & $\begin{array}{l}60 \\
50\end{array}$ & $\begin{array}{l}0.5 \\
0.0\end{array}$ & $\begin{array}{l}140 \\
140\end{array}$ & 1.5 & $\begin{array}{l}0.3 \\
05\end{array}$ & $\begin{array}{l}11.0 \\
120\end{array}$ & $\begin{array}{l}0.7 \\
0.5\end{array}$ & $\begin{array}{l}10 \\
00\end{array}$ & $\begin{array}{l}25 \\
30\end{array}$ & 3.5 & 5.0 & 10 & 20 & 0.5 & 05 & $\because 0$ & 0 & 00 & 0.0 & 0.3 & 1.5 & 0.5 & 10 & 00 & 0.0 & 0.0 & 0.0 \\
\hline & \multirow{6}{*}{ E. turriseiffelii } & $148-1,40-11$ & 41.0 & 5.0 & 1.0 & 18.0 & 1.5 & $\begin{array}{l}0.5 \\
0.5\end{array}$ & $\begin{array}{l}120 \\
130\end{array}$ & 0.5 & 1.5 & 30 & 30 & 4 & 15 & 0 & $\begin{array}{l}0.0 \\
00\end{array}$ & 00 & $\begin{array}{l}0.0 \\
00\end{array}$ & $0_{0}^{0}$ & : & $\begin{array}{l}0.0 \\
00\end{array}$ & 0.5 & 0 & $\begin{array}{l}0.5 \\
0.0\end{array}$ & $\begin{array}{l}0.5 \\
00\end{array}$ & & 0.0 & 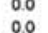 & 0.5 \\
\hline & & $16 R-1,20-21$ & 20.0 & 7.6 & 0.5 & 26.5 & 1.4 & 0.3 & 16.2 & 0.7 & 3.8 & 3.3 & 3.6 & 4.6 & 22 & 20 & 0.5 & 1.0 & 0.0 & 0 & 0.0 & 0.0 & 0.0 & 7 & 0.0 & 10 & 0.0 & 0.5 & $\begin{array}{l}1.0 \\
1.0\end{array}$ & 0.0 \\
\hline & & $177-1,0-1$ & 40 & 6.0 & 00 & 16.5 & 1.8 & 0.3 & 21.0 & 0.7 & 1.5 & 28 & 1.5 & 2.3 & 20 & 40 & 0.5 & 0.5 & $\therefore 0$ & 0 & 00 & 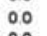 & 0.3 & & 0.5 & Sa & 5 & 3 & 2.5 & 5 \\
\hline & & $18 \mathrm{~A}-1,32-33$ & 18. & 7.4 & 0.5 & 20.0 & 1.7 & 1.9 & 6.7 & 0.5 & 0 & 22 & 8.4 & 8.1 & 1.7 & & 0.3 & 0 & 0 & & & & 0.3 & & 00 & & & .0 & 0.0 & \\
\hline & & $\begin{array}{l}19 R-1,65-67 \\
20 R-1,20-25\end{array}$ & $\begin{array}{l}\begin{array}{l}35.0 \\
31.0\end{array} \\
\end{array}$ & $\begin{array}{l}8.0 \\
11.7\end{array}$ & $\begin{array}{l}0.0 \\
0.0\end{array}$ & $\begin{array}{l}21.0 \\
24.7\end{array}$ & 100 & $\begin{array}{l}1.0 \\
1.0\end{array}$ & $\begin{array}{l}8.0 \\
102\end{array}$ & $\begin{array}{l}0.0 \\
0.0\end{array}$ & $\begin{array}{l}0.5 \\
0.6\end{array}$ & $\begin{array}{l}50 \\
58\end{array}$ & $\begin{array}{l}3.0 \\
3.8\end{array}$ & $\begin{array}{l}3.0 \\
2.3\end{array}$ & $\begin{array}{l}20 \\
23\end{array}$ & $\begin{array}{l}100 \\
0.8\end{array}$ & $\begin{array}{l}0.5 \\
0.8\end{array}$ & $\begin{array}{l}0.0 \\
0.8\end{array}$ & $\because 0$ & $\because 0$ & $\begin{array}{l}0.0 \\
0.3\end{array}$ & $\therefore$ & 00 & 10 & $\because 0$ & 00 & $\because 0$ & $\because 0$ & 0.0 & 0.0 \\
\hline & & $21 R-2,3$ & 31.0 & 120 & 00 & 200 & 1.5 & 10 & 12.0 & 0.0 & 0.5 & 30 & 10 & 25 & 1.5 & 10 & 00 & 00 & 00 & 00 & 00 & 00 & 00 & 00 & 00 & 00 & 00 & 00 & 00 & 000 \\
\hline & \multirow{3}{*}{ P. columnata } & $23 \mathrm{~A}-1,21 \cdot 22$ & 340 & 128 & 0.5 & 13.3 & 34 & 10 & 125 & 10 & 0.5 & 26 & 0.0 & 26 & 30 & 20 & 1.8 & 0.8 & 00 & 0.3 & 0.3 & 0.0 & 0.3 & 26 & 0.5 & 15 & 0.0 & 1.0 & 0.0 & 0.00 \\
\hline \multirow{7}{*}{$\frac{\Phi 5}{2}$} & & 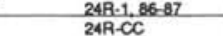 & $\frac{380}{310}$ & $\frac{10.3}{120}$ & $\frac{16}{40}$ & 4.19 & $\frac{57}{30}$ & $\frac{0.8}{15}$ & $\frac{62}{55}$ & $\frac{0.8}{00}$ & $\frac{0.5}{00}$ & $\frac{0.4}{15}$ & $\frac{00}{00}$ & $\frac{00}{00}$ & $\frac{3.7}{20}$ & $\frac{12}{20}$ & $\frac{28}{30}$ & $\frac{0.4}{30}$ & $\frac{00}{10}$ & $\frac{00}{00}$ & $\frac{0.8}{100}$ & $\frac{0.5}{00}$ & $\frac{00}{00}$ & 10 & $\frac{24}{0.5}$ & $\frac{0.4}{00}$ & 00 & $\frac{00}{00}$ & $\frac{12}{05}$ & $\frac{00}{00} \cong$ \\
\hline & & 2SR-CC & 300 & 140 & 40 & 50 & 3.0 & 3.0 & 60 & 10 & 00 & 10 & 00 & 00 & 20 & 20 & 3.5 & 30 & 10 & 03 & 100 & 10 & 0.5 & 20 & 1.0 & 10 & 0.0 & 1.0 & 0.5 & \\
\hline & \multirow{2}{*}{$P$. angustus } & $26 \mathrm{R}-1,18-20$ & 31.0 & 14.5 & 3.4 & 28 & 3.1 & 2.1 & 5.3 & 0.0 & 00 & 1.3 & 0.0 & 0.0 & 1.5 & 0 & 3.1 & 3.4 & 00 & 3 & 10.3 & 0.8 & 0.0 & is & 1.0 & 1.3 & 00 & 10 & 0.5 & 0.0 \\
\hline & & $\begin{array}{l}26 R-2.2103 \\
27 R_{1}\end{array}$ & $\begin{array}{l}32.0 \\
40\end{array}$ & $\begin{array}{l}12.6 \\
172\end{array}$ & 1.3 & 5.6 & $\begin{array}{l}5.4 \\
66\end{array}$ & 3.8 & $\begin{array}{l}3.5 \\
08\end{array}$ & $\begin{array}{l}0.5 \\
0.3\end{array}$ & $\because 0$ & 6.5 & $\because 0$ & $\therefore 0$ & 1.5 & $\because 0$ & 0.5 & 05 & $\therefore 0$ & 00 & 18.6 & 1.3 & 0.3 & 18 & 0.0 & 0.3 & 0.0 & 0.8 & 0.5 & 0.0 \\
\hline & \multirow{3}{*}{ C. litterarius } & $28 \mathrm{~A}-3,3,73-75$ & 38.0 & 6.3 & 1.3 & 76 & 2.8 & 2.1 & 5.3 & 1.3 & 00 & 6.5 & 00 & 0.0 & 0.0 & 2.1 & 0.8 & 10 & 0.3 & 0.5 & 16.0 & $\frac{10}{10}$ & 0.0 & 10 & 0.0 & 0.0 & 0.5 & 0.8 & 0.8 & 0.0 \\
\hline & & & 530 & 5.7 & 42 & 0.0 & 5.3 & 0.8 & 10 & 0.0 & 0.0 & 5.8 & 0.0 & 0. & 1.8 & 0.0 & 29 & 1.3 & 29 & 00 & 7.6 & 4 & & & 0.0 & 00 & & 0 & 10 & 0.0 \\
\hline & & $\begin{array}{l}37 A-C C \\
38 A-1,100\end{array}$ & $\begin{array}{l}55.0 \\
55.0\end{array}$ & $\begin{array}{l}6.0 \\
6.0\end{array}$ & $\begin{array}{l}40 \\
4.0\end{array}$ & $\begin{array}{l}0.5 \\
10\end{array}$ & $\begin{array}{l}5.0 \\
5.0\end{array}$ & $\begin{array}{l}0.5 \\
1.0\end{array}$ & $\begin{array}{l}100 \\
10\end{array}$ & $\begin{array}{l}0.0 \\
0.0\end{array}$ & $\begin{array}{l}00 \\
0.0\end{array}$ & $\begin{array}{l}40 \\
5.0\end{array}$ & $\begin{array}{l}0.0 \\
0.0\end{array}$ & $\begin{array}{l}0.0 \\
0.0\end{array}$ & $\begin{array}{l}20 \\
2.0\end{array}$ & $\begin{array}{l}0.0 \\
0.0\end{array}$ & $\begin{array}{l}20 \\
1.5\end{array}$ & $\begin{array}{l}1.0 \\
2.0\end{array}$ & $\begin{array}{l}20 \\
20\end{array}$ & $\begin{array}{l}0.0 \\
0.0\end{array}$ & $\begin{array}{l}8.0 \\
6.0\end{array}$ & $\begin{array}{l}4.0 \\
3.0\end{array}$ & $\begin{array}{l}0.0 \\
0.0\end{array}$ & 10 & $\begin{array}{l}0.0 \\
0.0\end{array}$ & $\begin{array}{l}0.0 \\
0.0\end{array}$ & $\begin{array}{l}0.5 \\
1.0\end{array}$ & $\begin{array}{l}0.5 \\
0.0\end{array}$ & $\begin{array}{l}0.0 \\
0.0\end{array}$ & $\begin{array}{l}0.0 \\
0.0\end{array}$ \\
\hline \multirow{10}{*}{ 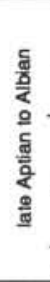 } & \multirow{4}{*}{ E. turriseiffelii } & $120-801 \mathrm{~A}-14 \mathrm{R}, \propto C$ & 41.0 & 162 & 0.3 & 6.3 & 9.5 & 3.5 & 8.4 & 0.8 & 1.5 & 0.3 & 1.7 & 0.3 & 1.5 & 0.0 & 0.5 & 0.0 & 0.0 & 00 & 0.0 & 0.0 & 0.8 & 50 & 0.0 & 0.0 & 0.8 & 0.5 & 0.0 & 0.0 \\
\hline & & 15 & 40.0 & 77 & 0.0 & 19.5 & 1.9 & 0.3 & 134 & 0.0 & 0.5 & (1.8 & 42 & 3 & 0.5 & 10 & 0.3 & 100 & 00 & 0.0 & 00 & 0. & 0.0 & 0.3 & 0.0 & 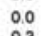 & 年 & 10 & 0.0 & 0 \\
\hline & & & 18.4 & 62 & 0.3 & 247 & 2.1 & 0.3 & & 0 & 4. & 1.6 & & & 0.3 & & & & & & 00 & 0.0 & & 1.3 & 0.0 & 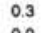 & 0.0 & 0.3 & 0.0 & \\
\hline & & $\begin{array}{l}7 A-1.2 .5 \\
19 R \cdot-1,43\end{array}$ & $\begin{array}{l}17.0 \\
26.0\end{array}$ & $\begin{array}{l}8.0 \\
95\end{array}$ & $\begin{array}{l}0.0 \\
03\end{array}$ & $\begin{array}{l}257 \\
257\end{array}$ & $\begin{array}{l}10 \\
29\end{array}$ & $\begin{array}{l}2.8 \\
0.5\end{array}$ & $\begin{array}{l}300 \\
142\end{array}$ & $\begin{array}{l}0.8 \\
03\end{array}$ & $\begin{array}{l}28 \\
20\end{array}$ & 87 & $\begin{array}{l}2.2 \\
2.6\end{array}$ & 18 & $\begin{array}{l}0.8 \\
0.8\end{array}$ & $\begin{array}{l}1.9 \\
0.0\end{array}$ & $\begin{array}{l}0.3 \\
0.0\end{array}$ & $\begin{array}{l}0.0 \\
0.0\end{array}$ & $\begin{array}{l}0.0 \\
0.0\end{array}$ & $\begin{array}{l}0.0 \\
00\end{array}$ & $\begin{array}{l}0.0 \\
00\end{array}$ & $\begin{array}{l}0.0 \\
0.0\end{array}$ & $\begin{array}{l}0.0 \\
0.0\end{array}$ & 1.0 & 2.2 & 0.0 & 0.0 & 0.00 & 0.0 & $\begin{array}{l}0.0 \\
0.0\end{array}$ \\
\hline & \multirow{6}{*}{ P. columnata } & $120-8018-18-1,59-60$ & 30.0 & 11.0 & 1.0 & 14.0 & 20 & 0.5 & 120 & 0.0 & 0.5 & 70 & 0.0 & 0.5 & 10 & 0.5 & 0.0 & 0.0 & 0.0 & 0.0 & 00 & 0.0 & 0.0 & 1.0 & 0.0 & 0.0 & 0.0 & 0.0 & 0.0 & 0.0 . \\
\hline & & & 30.0 & 11.0 & 0.5 & 15.0 & 40 & 1.0 & 140 & 0.0 & 0.0 & 60 & 0.0 & 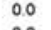 & 10 & 0 & & 00 & & & 0.5 & 0.0 & & & م0 & 0 & & 05 & 0.0 & $\Phi$ \\
\hline & & $\begin{array}{l}6 R-1,109-110 \\
6 \text { GR-2, 36 }\end{array}$ & $\begin{array}{l}28.0 \\
21.0\end{array}$ & $\begin{array}{l}111.1 \\
12.2\end{array}$ & $\begin{array}{l}0.0 \\
0.0\end{array}$ & $\begin{array}{l}16.5 \\
14.8\end{array}$ & $\begin{array}{l}75 \\
7.1\end{array}$ & $\begin{array}{l}22 \\
10\end{array}$ & $\begin{array}{l}162 \\
14.8\end{array}$ & 100 & $\begin{array}{l}10.0 \\
0.5\end{array}$ & $\begin{array}{r}58 \\
174\end{array}$ & 0.0 & $\begin{array}{l}0.0 \\
0.0\end{array}$ & $\begin{array}{l}0.3 \\
18\end{array}$ & 1.4 & $\begin{array}{l}0.5 \\
0.7\end{array}$ & 0.0 & $\begin{array}{l}0.0 \\
00\end{array}$ & $\begin{array}{l}0.0 \\
00\end{array}$ & $\begin{array}{l}1.9 \\
00\end{array}$ & $\begin{array}{l}0.5 \\
0.3\end{array}$ & $\begin{array}{l}0.3 \\
03\end{array}$ & $\begin{array}{l}1.6 \\
25\end{array}$ & 0.5 & 0.8 & 0.0 & 14 & 0.3 & 0.0 \\
\hline & & $\begin{array}{l}6 A-2,36 \\
6 A-3,10-11\end{array}$ & 20.0 & 82 & 00 & 15.2 & 82 & 08 & 167 & 05 & 0.8 & 18.5 & 0.0 & 0.0 & 08 & 1.5 & 0.3 & 00 & 0.0 & 0.0 & 1.5 & 0.0 & 0.5 & $\begin{array}{l}2.8 \\
0.8\end{array}$ & 10 & 0.3 & 0.0 & 0.8 & 05 & 0.0 \\
\hline & & 7R.1. 6 & 280 & 8.8 & 00 & 13.3 & 5.5 & 12 & 160 & 0.3 & 10 & 200 & 0.0 & 0.0 & 0.6 & 0.3 & 00 & 00 & 0.3 & 00 & 12 & 00 & 00 & 0.0 & 00 & 06 & 0.0 & 12 & 12 & 00 \\
\hline & & 8R-4, 111-112 & 27.0 & 8.0 & 0.5 & 13.0 & 5.0 & 10 & 16.0 & 0.0 & 0.0 & 18.0 & 0.0 & 0.0 & 05 & 0.0 & 0.0 & 0.0 & 0.5 & 0.0 & 0.5 & 0.5 & 0.0 & 0.5 & 0.5 & 0.5 & 0.5 & 0.5 & 0.5 & 0.0 \\
\hline \multirow{3}{*}{ 轺 } & \multirow[t]{4}{*}{12} & $120-802 A-50$ & 58 & 148 & 0 & 77 & 5 & 0 & 1.7 & 00 & 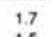 & 25 & & & 0.8 & ( & & $\circ$ & 00 & & 2 & 50 & 0 & 1. & 00 & 0.0 & 0 & 00 & 0.0 & \\
\hline & & & 550 & 15.0 & 0.0 & 30 & 5 & 0 & 25 & 00 & 1. & 40 & & & 0 & 0 & & & & & & 1.5 & & & & & & & & \\
\hline & & $\begin{array}{l}\text { SARR }-C \text {. } \\
\text { S5F-1,16 }\end{array}$ & $\begin{array}{l}50.0 \\
53.0\end{array}$ & $\begin{array}{l}14.0 \\
20.1\end{array}$ & $\begin{array}{l}1.0 \\
0.0\end{array}$ & $\begin{array}{l}4.0 \\
4.2\end{array}$ & $\begin{array}{l}8.0 \\
14.3\end{array}$ & $\begin{array}{l}0.5 \\
0.0\end{array}$ & $\begin{array}{l}1.0 \\
0.9\end{array}$ & $\begin{array}{l}0.0 \\
0.0\end{array}$ & $\begin{array}{l}1.0 \\
0.0\end{array}$ & $\begin{array}{l}3.0 \\
0.3\end{array}$ & $\begin{array}{l}0.5 \\
1.3\end{array}$ & $\begin{array}{l}0.5 \\
0.0\end{array}$ & $\begin{array}{l}0.5 \\
0.0\end{array}$ & $\begin{array}{l}0.0 \\
0.0\end{array}$ & $\begin{array}{l}0.0 \\
0.0\end{array}$ & $\begin{array}{l}0.0 \\
0.0\end{array}$ & $\begin{array}{l}0.0 \\
0.0\end{array}$ & $\begin{array}{l}0.0 \\
0.0\end{array}$ & $1.62-3>$ & $\begin{array}{l}0.5 \\
0.0\end{array}$ & $\begin{array}{l}0.5 \\
1.0\end{array}$ & 1 & $\begin{array}{l}0.5 \\
0.0\end{array}$ & 0.6 & $\begin{array}{l}0.5 \\
1.0\end{array}$ & $\begin{array}{l}0.0 \\
0.3\end{array}$ & $\begin{array}{ll}0.0 \\
00\end{array}$ & $0.0 \%$ \\
\hline & & SSR-CC & $\frac{540}{550}$ & $\frac{190}{180}$ & 0.5 & 40 & 140 & 00 & 10 & es & 00 & 20 & 00 & 0.5 & 20 & 20 & 05 & 00 & Q5 & 00 & 15 & 20 & 50 & ses & 00 & $x_{0}$ & 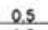 & 05 & 00 & 00 \\
\hline & Not zoned & $57 R-1,84-85$ & & & & & 140 & & & & & & & & & & & & & & & & & & & & & 0.5 & 0.0 & \\
\hline
\end{tabular}




\section{LEG 62 - SITE 463}

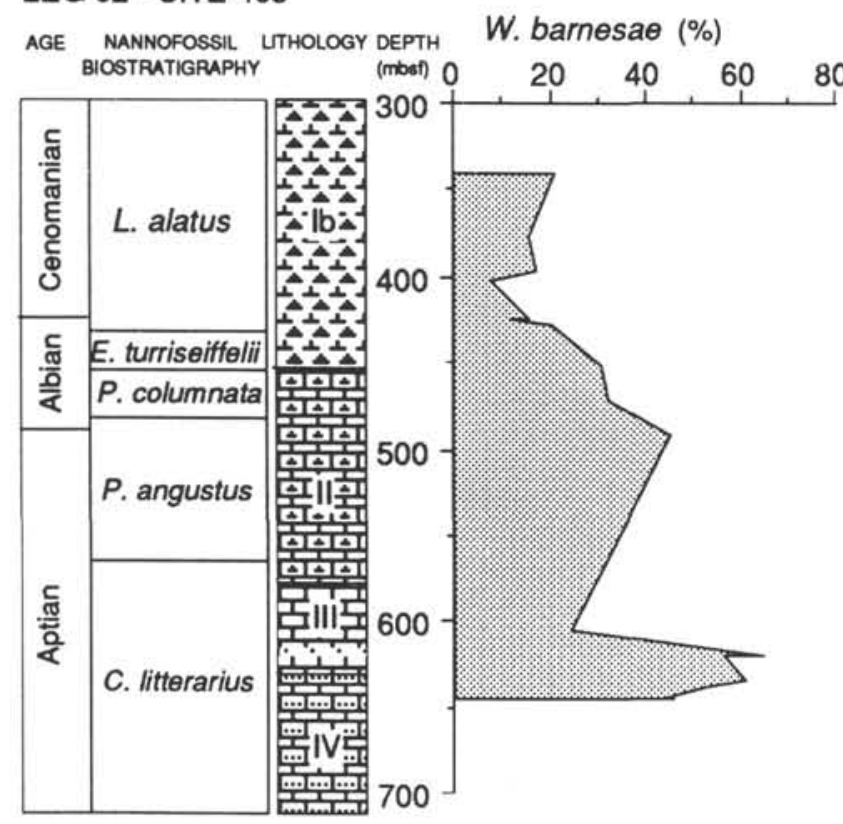

NANNOFOSSIL HIGH-FERTILITY INDICES

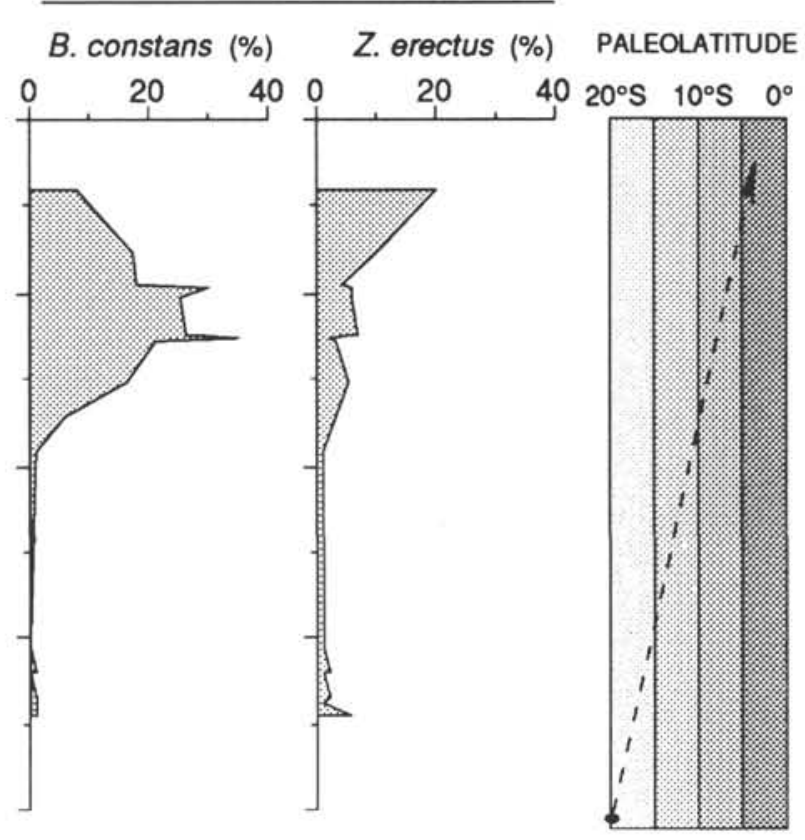

Figure 3. Distribution of the fertility indicators in the Aptian-Cenomanian interval at Site 463 (modified after Roth, 1981). The paleolatitude plot, suggesting a motion toward the paleoequator, is inferred from Thiede et al. (1981).

$R$. irregularis is relatively abundant in the upper lower and upper Aptian and sharply decreases in the Albian. Peaks in abundance of this species were noted in coeval samples of other sedimentary sequences (Erba, 1986, in press; T. Bralower, pers. comm., 1991) and might be induced by a paleoceanographic event on a global scale. $R$. irregularis is particularly abundant at low latitudes and might be an index of warmer waters. However, fluctuations in abundance of this species at low latitudes are not clearly related to changes of the surface water temperature and consequently the paleoecologic affinities of $R$. irregularis remains unrecognized.

In Sample 129-800A-26R-2, $103 \mathrm{~cm}$, a relative increase in the abundance of Braarudosphaera africana, which record a value of $3 \%$, was observed (Fig. 5). This species is not listed in Table 2 because it is absent in all other selected samples.

The most striking change in nannofossil assemblages is recorded in the middle Albian, where the abundance of the high-fertility indicators $B$. constans and $Z$. erectus are at least twice as high than in older samples: they increase from values of approximately $5 \%$ to values of more than $20 \%$ and both species reach maximum values in the $E$. turriseiffelii Zone. It must be noted however, that $B$. constans reaches the highest abundance value (Core 129-800A-18R) slightly before Z. erectus (Core 129-800A-17R).

In Figure 6 the changes in abundance of the nannofossil fertility indices are compared with semiquantitative data on radiolarian abundance ("Biostratigraphy" section, "Site 800" chapter, Lancelot, Larson, et al., 1990) and with paleolatitudes computed from mean inclination measured in the sediments ("Paleomagnetics" section, "Site 800" chapter, Lancelot, Larson, et al., 1990).

An interval of extreme nannofossil dissolution occurs in the lower Aptian (C. litterarius nannofossil Zone). It corresponds to an interval with peaks in abundance of radiolarians that are otherwise absent or rare in the Aptian of this site. This change in planktonic assemblages is most probably not diagenetically induced; in fact, in the upper lower Aptian the few pelagic intervals recovered consist of porcellanites, whereas older and younger pelagic sediments within the Aptian are nannofossil limestone and claystone. During the Aptian, Site 800 moved from a paleolati- tude of $14^{\circ} \mathrm{S}$ to $10^{\circ} \mathrm{S}$ and changes in planktonic assemblages are not correlatable to significant changes in paleolatitude. Peculiarly, the sharp decrease in abundance of calcareous nannofossils coinciding with the peak of radiolarians observed in the upper lower Aptian at Site 800 resemble coeval records from the Pacific ocean (Roth, 1981; Sliter, 1989) and from the Tethyan area, where a widespread "black shale" event, named "Selli Level," was recognized (Wezel, 1985; Coccioni et al., 1989; Erba, 1986, in press). The Selli Level black shale, barren of calcareous plankton and characterized by extremely abundant radiolarians, has been interpreted as a high-productivity event probably triggered by a global change in the middle Cretaceous ocean. Recently Larson (1991a, 1991b) documented the eruption of a superplume taking place in the early Aptian in the Pacific basin. This pulse in oceanic crust production is correlatable with the onset of a number of peculiar mid-Cretaceous events, such as the long interval of normal magnetic polarity, black shale deposition, increase of world temperature, rise in eustatic sea level, a carbonate dissolution event (Larson, 1991b). Major changes in nannofossil assemblage composition and taxonomic diversification have been correlated to this superplume eruption (Erba and Larson, 1991).

The changes in the planktonic communities in the upper lower Aptian at Site 800 might be correlatable to this superplume and represent the response to the new oceanic environment characterized by a high intraplate volcanism. A possible link between volcanic episodes and deposition of carbonaceous sediments was suggested also by Schlanger et al. (1987) for the mid-Cretaceous black shales.

At Site 800 the shift of nannofloras to high-fertility assemblages occurs in the middle Albian and is accompanied by an increase in abundance of radiolarians (Fig. 6). At this time Site 800 records a paleolatitude close to $7^{\circ} \mathrm{S}$. During the rest of the AlbianCenomanian nannofloras remain abundant and characterized by high percentages of both $B$. constans and $Z$. erectus. These two species reach maximum abundance in Cores $129-800 \mathrm{~A}-18 \mathrm{R}$ and 129-800A-17R, respectively, where radiolarians record a major increase in abundance as well and paleolatitude values are close to $5^{\circ} \mathrm{S}$. 
Another major change occurs in Core $129-800 \mathrm{~A}-10 \mathrm{R}$, which is barren of nannofossils and where radiolarians are the only biogenic components of the sediments. Paleolatitude was close to $2^{\circ} \mathrm{S}$ suggesting that Site 800 was very close to the paleoequator and sedimentation was taking place in the inner part of the upwelling belt.

\section{Site 801}

At Site 801 only the uppermost Aptian and Albian sediments contain calcareous nannofossils. In this interval the assemblages are characterized by high abundances of both $B$. constans and $Z$. erectus (Table 2 and Fig. 7), with values very similar to those recorded for the middle Albian to Cenomanian interval of Site 800. These data suggest proximity to the upwelling equatorial belt.

A decrease in abundance of both nannofossils and radiolarians in the lower to middle Albian (Cores 129-801B-4R to 129-801B-2R) (Fig. 8) is due to coarser volcaniclastic deposits with no pelagic interbeds.

$B$. constans increases in abundance in the lower part of the E. turriseiffelii Zone (Sample 129-801A-19R-1, $43 \mathrm{~cm}$ ) passing from values of approximately $15 \%$ to values of approximately $25 \%$. The increase in abundance of $Z$. erectus is slightly younger than that of $B$. constans. Values close to $30 \%$ are recorded for $Z$. erectus in Cores 129-801A-17R and 129-801A-16R, whereas percentages of approximately $15 \%$ were recorded in older samples. This pattern, where $B$. constans increases in abundance prior to $Z$. erectus, is consistent with observations at Site 800 and data reported by Roth (1981) at Site 463 (Fig. 3). Although both species have been interpreted as indicators of high surface-water fertility, discrepancies in their distribution were noted (Erba et al., 1989; Erba, Castradori, Guasti, and Ripepe, in press; Roth, 1989; Watkins, 1989; Thierstein and Roth, 1991). From the present study it seems that $Z$. erectus becomes really abundant only in the upwelling belt, whereas $B$. constans increases in abundance already at the margins of the high-fertility area. If this is correct, $B$. constans is sensitive to high fertility in a mesotrophic environment, whereas $Z$. erectus is an index of higher fertility in a more eutrophic environment. In passing to a highly eutrophic environment nannofossils are first affected by dissolution and then totally replaced by siliceous plankton. In fact, calcareous nannofossil assemblages record a relative decrease in abundance of the fertility indicators species in Core 129-801A-14R, and sediments above Core 129-801A-13R are barren of nannofossils. These changes in the nannofloras are accompanied by a sharp increase in abundance of radiolarians, which become the only biogenic component in Cenomanian and younger sediments. As shown in Figure 8, changes of planktonic assemblages are correlatable to paleolatitudes computed from mean inclination measured in the sediments ("Paleomagnetism" section, "Site 801" chapter, Lancelot, Larson, et al., 1990). Calcareous nannofloras were most abundant and characterized by the maximum values of the high-fertility indices when Site 801 was at paleolatitudes between $10^{\circ} \mathrm{S}$ and $5^{\circ} \mathrm{S}$. At the same time radiolarians experienced an increase in abundance. When paleolatitudes were closer to the paleoequator (approximately $2^{\circ} \mathrm{S}$ ) nannofossils disappeared and radiolarians dominated the upwelling area. These results are consistent with those reported for Site 800 .

In the undated Valanginian to upper Aptian interval recorded at Site 801 calcareous nannofossils are absent, but radiolarians show a peak in abundance in samples just below the upper Aptian sediments. This short interval might correspond to the dissolution event recorded in the upper lower Aptian at Site 800.

\section{Site $\mathbf{8 0 2}$}

Only a few samples from the middle Cretaceous of Site 802 were selected for counting of nannofossils. In fact, although abundant, nannofossil assemblages are often poorly preserved and $W$. barnesae constitutes more than $50 \%$ of nannofloras (Table 2 and Fig. 9). In the studied samples
B. constans and $\mathrm{Z}$ erectus are common but never really abundant. Throughout this interval radiolarians are very abundant and become the only biogenic component of pelagic sediments from Core 129-802A$52 \mathrm{R}$, where nannofossils disappear. Paleolatitude values computed from mean inclination measured in the sediments ("Paleomagnetism" section, "Site 802" chapter, Lancelot, Larson, et al., 1990) indicate the motion of Site 802 toward the paleoequator, but values of approximately $8.5^{\circ} \mathrm{S}$ to $7^{\circ} \mathrm{S}$ were measured for this interval (Fig. 10). Nannofossil assemblages might reflect a poorer preservation in a deep environment or moderate fertility composition.

Paleolatitudes were much closer to the paleoequator in Santonian and Campanian times but nannofossil assemblages were not studied because the paleoecology of the Late Cretaceous nannofloras is not known yet.

\section{DISCUSSION AND CONCLUSIONS}

Quantitative data of calcareous nannofossils from middle Cretaceous sediments recovered during Leg 129 display changes in assemblage composition. A careful selection of pelagic layers was performed to analyze only the best preserved nannofloras. Changes of nannofossil assemblages are recorded mainly by $B$. constans and $Z$. erectus displaying wide fluctuations of relative abundance. In previous studies of middle Cretaceous paleoceanography, these species were recognized as high-fertility indicators sharply increasing in abundance in the paleoequatorial belt of the central Pacific and upwelling sites from the Atlantic Ocean and other marginal seas. Integration of the nannofossil data with radiolarian distribution and paleolatitude values calculated from sediment inclination, contributes to reconstructing the motion of the Pacific plate with respect to the paleoequator.

At Sites 800 and 801 , the high-fertility indices sharply increase in abundance in proximity to the southern edge of the paleoequatorial belt (approximately $7^{\circ}$ to $5^{\circ} \mathrm{S}$ ). When sites reached the core of the upwelling zone (approximately $2^{\circ} \mathrm{S}$ ), nannofossils were totally replaced by radiolarians. Moreover, at both sites, B. constans increases in abundance prior to Z. erectus as also documented by Roth (1981) at Site 463 . Therefore, it seems that $Z$. erectus becomes really abundant only in the upwelling belt, whereas $B$. constans increases in abundance already at the margins of the high-fertility area. If this is correct, B. constans is sensitive to high fertility in a mesotrophic environment, whereas $Z$. erectus is an index of higher fertility in a more eutrophic environment. In passing to a highly eutrophic environment nannofossils are first affected by dissolution and then totally replaced by siliceous plankton. This hypothesis is illustrated in Figure 11, where Cretaceous planktonic microfossils are tentatively compared to the surface water trophic conditions. The upper part of Figure 11 represents the oceanic surface-water trophic-resource continuum (TRC), comparing the modern Atlantic and Pacific oceans.

The studies conducted on the modern nannoplankton in the Pacific Ocean (Okada and Honjo, 1973; Roth and Berger, 1975; Geitzenauer et al., 1976; Roth and Coulbourn, 1982) show latitudinal patterns in the nannofloral composition besides a strong influence of preservation. However, selective dissolution only partially obscures the record of coccolith provinciality: an equatorial assemblage was detected and related to higher nutrient content and relatively cooler temperature in the equatorial upwelling belt. Likewise, the nannofloral distribution in the Cretaceous Pacific ocean seem to reflect the more fertile conditions of the paleoequatorial upwelling area.

At Site 802 nannofossils are abundant but poorly preserved. B. constans and $Z$. erectus are not abundant throughout the middle Cretaceous. The assemblages might reflect sedimentation at a site south of the paleoequatorial divergence but could also be the result of increased dissolution in a deeper environment.

Adissolution event was observed in upper lower Aptian sediments from Site 800 . Nannofossils are virtually absent and the radiolarians record a peak in abundance while they were not observed in the lower and upper Aptian. Similar patterns were recognized in coeval se- 
quences from the Pacific ocean and the Tethyan area and might be triggered by a global oceanic event such as the intraplate volcanic activity recently documented by Larson (1991a, 1991b).

\section{ACKNOWLEDGMENTS}

I wish to express my gratitude to Yves Lancelot and Roger Larson for their enthusiastic leadership, which made Leg 129 a wonderful, unforgettable adventure. I greatly benefited from their advice and support during the cruise and during shore-based studies as well. Sincere thanks are extended to Leg 129 scientific party for discussion and cooperation.

Hans Thierstein and Katharina von Salis Perch-Nielsen are acknowledged for constructive suggestions and critical review of the manuscript. Research was supported by CNR funds.

\section{REFERENCES}

Bown, P. R., 1987. Taxonomy, evolution and biostratigraphy of Late TriassicEarly Jurassic calcareous nannofossils. Spec. Pap. Paleontol., 38:1-118.

Bralower, T. J., 1987. Valanginian to Aptian calcareous nannofossil stratigraphy and correlation with the upper M-sequence magnetic anomalies. Mar. Micropaleontol., 11:293-310.

Bralower, T. J., Bown, P. R., and Siesser, W. G., 1991. Significance of Upper Triassic nannofossils from the Southern Hemisphere (ODP Leg 122 , Wombat Plateau, N.W. Australia). Mar. Micropaleontol., 17:119-154.

Caron, M., and Homewood, P., 1983. Evolution of early planktic foraminifers. Mar. Micropaleontol., 7:453-462.

Coccioni, R., Franchi, R., Nesci, O., Wezel, C. F., Battistini, F., and Pallecchi, P., 1989. Stratigraphy and mineralogy of the Selli Level (early Aptian) at the base of the Marne a Fucoidi in the Umbrian-Marchean Apennines (Italy). In Wiedmann, J. (Ed.), Cretaceous of the Western Tethys: Proc. 3rd Int. Cretaceous Symp., Tubingen 1987, E. Schweizerbart'sche Verlagsbuchhandlung, 230-245.

Erba, E., 1986. I Nannofossili calcarei nell'Aptiano-Albiano (Cretacico inferiore): biostratigrafia, paleoceanografia e diagenesi degli Scisti a Fucoidi del pozzo Piobbico (Marche) [Ph.D. dissert.]. Milano Univ.

, 1987. Mid-Cretaceous cyclic pelagic facies from the UmbrianMarchean Basin: what do calcareous nannofossils suggest? INA Newsl, 9:52-53.

, in press. Calcareous nannofossil distribution in pelagic rhythmic sediments (Aptian-Albian Piobbico core, Central Italy). Riv. Ital. Paleontol. Stratigr., 97.

Erba, E., Castradori, D., and Cobianchi, M., in press. Compilation of Late Triassic and Jurassic calcareous nannofossil ranges. Mem. Sci. Geol. Padova, 43:27-40.

Erba, E., Castradori, D., Guasti, G., and Ripepe, M., in press. Calcareous nannofossils and Milankovitch cycles: the example of the Albian Gault Clay Formation (southern England). Palaeogeogr., Palaeoclimatol., Palaeoecol.

Erba, E., Guasti, G., and Castradori, D., 1989. Calcareous nannofossils record fertility and temperature cycles: Evidence from the Albian Gault Clay Formation. INA Newsl., 11:57-58.

Erba, E., and Larson, R. L., 1991. Nannofossils and superplumes. Eos, Abstract Volume for AGU Spring Meeting-Baltimore 1991, 301.

Geitzenauer, K. R., Roche, M. B., and McIntyre, A., 1976. Modern Pacific coccolith assemblages: derivation and application to late Pleistocene paleotemperature analyses. Geol. Soc. Am. Mem., 145:423-448.

Hallock, P., 1987. Fluctuations in the trophic resource continuum: A factor in global diversity cycles? Paleoceanography, 2:457-471.

Kent, D. V., and Gradstein, F. M., 1985. A Cretaceous and Jurassic geochronology. Geol. Soc. Am. Bull., 96:1419-1427.

Lancelot, Y., 1978. Evolution sedimentaire et tectonique de la plaque Pacifique. Mem. Soc. Geol. Fr., Nelle Ser., 134.

Lancelot, Y., and Larson, R. L., 1975. Sedimentary and tectonic evolution of the northwestern Pacific. In Larson, R. L., Moberly, R., et al., Init. Repts. DSDP, 32: Washington (U.S. Govt. Printing Office), 945-957.

Lancelot, Y., Larson, R. L., et al., 1990. Proc. ODP, Init. Repts., 129: College Station, TX (Ocean Drilling Program).

Larson, R. L., 1991a. Latest pulse of the Earth: evidence for a mid-Cretaceous superplume. Geology, 19:547-550.

19:9 191b. Geological consequences of superplumes. Geology, 19:963-966.
Leckie, R. M., 1987. Paleoecology of mid-Cretaceous planktonic foraminifera: a comparison of open ocean and epicontinental sea assemblages. Micropaleontology, 33:164-176.

,- 1989 . An oceanographic model for the early evolutionary history of planktonic foraminifera. Palaeogeogr., Palaeoclim., Palaeoecol., 73:107-138.

Monechi, S., and Thierstein, H. R., 1985. Late Cretaceous-Eocene nannofossil and magnetostratigraphic correlations near Gubbio, Italy. Mar. Micropaleontol., 9:419-440.

Mutterlose, J., 1987. Calcareous nannofossils and belemnites as warmwater indicators from the NW-German middle Aptian. Geol. Jahrb., 96:293-313. 1989. Temperature-controlled migration of calcareous nannofloras in the north-west European Aptian. In Crux, J. A., and van Heck, S. E. (Eds.), Nannofossils and Their Applications: Chichester (Ellis Horwood), Brit. Micropaleontol. Soc. Ser., 122-142.

, 1991. Das Verterilungs- und Migrations-Muster des kalkigen Nannoplanktons in der Unterkreide (Valangin-Apt) NW-Deutschland. $\mathrm{Pa}$ laeontographica B, 221:27-152.

Okada, H., and Honjo, S., 1973. The distribution of oceanic coccolithophorids in the Pacific. Deep-sea Res. Part A, 20:355-374.

Premoli Silva, I., Erba, E., and Tornaghi, M. E., 1989. Paleoenvironmental signals and changes in surface fertility in Mid Cretaceous $\mathrm{C}_{\text {org }}$-rich pelagic facies of the Fucoid Marls (Central Italy). Geobios, 11:225-236.

Roth,P. H., 1978. Cretaceous nannoplankton biostratigraphy and oceanography of the northwestern Atlantic Ocean. In Benson, W. E., Sheridan, R. E., et al., Init. Repts. DSDP, 44: Washington (U.S. Govt. Printing Office), 731-759.

1981. Mid-Cretaceous calcareous nannoplankton from the central Pacific: implication for paleoceanography. In Thiede, J., Vallier, T. L., et al., Init. Repts. DSDP, 62: Washington (U.S. Govt. Printing Office), $471-489$.

, 1983. Jurassic and Lower Cretaceous calcareous nannofossils in the Western North Atlantic (Site 534): biostratigraphy, preservation, and some observations on biogeography and paleoceanography. In Sheridan, R. E., Gradstein, F. M., et al., Init. Repts. DSDP, 76: Washington (U.S. Govt. Printing Office), 587-621.

1984. Preservation of calcareous nannofossils and fine-grained carbonate particles in mid-Cretaceous sediments from the southern Angola Basin. In Hay, W. W., Sibuet, J. C., et al., Init. Repts. DSDP, 75 (Pt. 2): Washington (U.S. Govt. Printing Office), 651-655.

1986. Mesozoic palaeoceanography of the North Atlantic and Tethys Oceans. In Summerhayes, C. P., and Shackleton, N. J. (Eds.), North Atlantic Palaeoceanography. Geol. Soc. Spec. Publ. London, 21:299-320. 1989. Ocean circulation and calcareous nannoplankton evolution during the Jurassic and Cretaceous. Palaeogeogr., Palaeoclimatol., Palaeoecol., 74:111-126.

Roth, P. H., and Berger, W. H., 1975. Distribution and dissolution of coccoliths in the south and central Pacific. In Sliter, W. V., Bé, A.W.H., and Berger, W. H. (Eds.), Dissolution of Deep-sea Carbonates. Spec. Publ. Cushman Found. Foraminiferal Res., 13:87-113.

Roth, P. H., and Bowdler, J. L., 1981. Middle Cretaceous calcareous nannoplankton biogeography and oceanography of the Atlantic ocean. In Warme, J. E., Douglas, R. G., and Winterer, E. L. (Eds.), The Deep Sea Drilling Project: a Decade of Progress, Spec. Publ.-Soc. Econ. Paleontol. Mineral., 32:517-546.

Roth, P. H., and Coulbourn, W. T., 1982. Floral and solution pattern of coccoliths in surface sediments of the North Pacific. Mar. Micropaleontol., 7:1-52.

Roth, P. H., and Krumbach, K. R., 1986. Middle Cretaceous calcareous nannofossil biogeography and preservation in the Atlantic and Indian oceans: implications for paleoceanography. Mar. Micropaleontol., 10:235-266.

Schlanger, S. O., Arthur, M. A., Jenkyns, H. C., and Scholle, P. A., 1987. The Cenomanian-Turonian Oceanic Anoxic Event, I. Stratigraphy and distribution of organic carbon-rich beds and the marine $\delta^{13} \mathrm{C}$. In Brooks, J., and Fleet, A. J. (Eds.), Marine Petroleum Source Rocks. Geol. Soc. Spec. Publ. London, 26:371-399.

Sissingh, W., 1977. Biostratigraphy of Cretaceous calcareous nannoplankton. Geol. Mijnbouw, 56:37-65.

Sliter, W. V., 1989. Aptian anoxia in the Pacific Basin. Geology, 17:909-910.

Sliter, W. V., and Premoli Silva, I., 1990. Age and origin of Cretaceous planktonic foraminifers from limestone of the Franciscan Complex near Laytonville, California. Paleoceanography, 5:639-667.

Thiede, J., Dean, W. E., Rea, D. K., Vallier, T. L., and Adelseck, C. G., 1981. The geologic history of the Mid-Pacific Mountains in the central North 
Pacific Ocean. A synthesis of deep-sea drilling studies. In Thiede, J., Vallier, T. L., et al., Init. Repts. DSDP, 62: Washington (U.S. Govt. Printing Office), 1073-1120.

Thierstein, H. R., 1971. Tentative Lower Cretaceous calcareous nannoplankton zonation. Eclogae Geol. Helv., 64:459-488.

1973. Lower Cretaceous calcareous nannoplankton biostratigraphy. Abh. Geol. Bundesanst. Austria, 29:1-52.

, 1976. Mesozoic calcareous nannoplankton biostratigraphy of marine sediments. Mar. Micropaleontol., 1:325-362.

1980. Selective dissolution of Late Cretaceous and earliest Tertiary calcareous nannofossils: experimental evidence. Cretaceous Res., 2:2-12.

1981. Late Cretaceous nannoplankton and the change at the Cretaceous-Tertiary boundary. In Warme, J. E., Douglas, R. G., and Winterer, E. L. (Eds.), The Deep Sea Drilling Project: a Decade of Progress. Spec. Publ.-Soc. Econ. Paleontol. Mineral., 32:355-394.
Thierstein, H. P., and Roth, P. H., 1991. Stable isotopic and carbonate cyclicity in Lower Cretaceous deep-sea sediments: dominance of diagenetic effects. Mar. Geol., 97:1-34.

Watkins, D. K., 1986. Calcareous nannofossil paleoceanography of the Cretaceous Greenhorn Sea. Geol. Soc. Am. Bull., 97:1239-1249.

1989. Nannoplankton productivity fluctuations and rhythmicallybedded pelagic carbonates of the Greenhorn Limestonen (Upper Cretaceous). Palaeogeogr., Palaeoclim., Palaeoecol., 74:75-86.

Wezel, C. F., 1985. Facies anossiche ed episodi geotettonici globali. G. Geol., $47: 281-286$

Date of initial receipt: 30 May 1991

Date of acceptance: 17 March 1992

Ms 129B-119

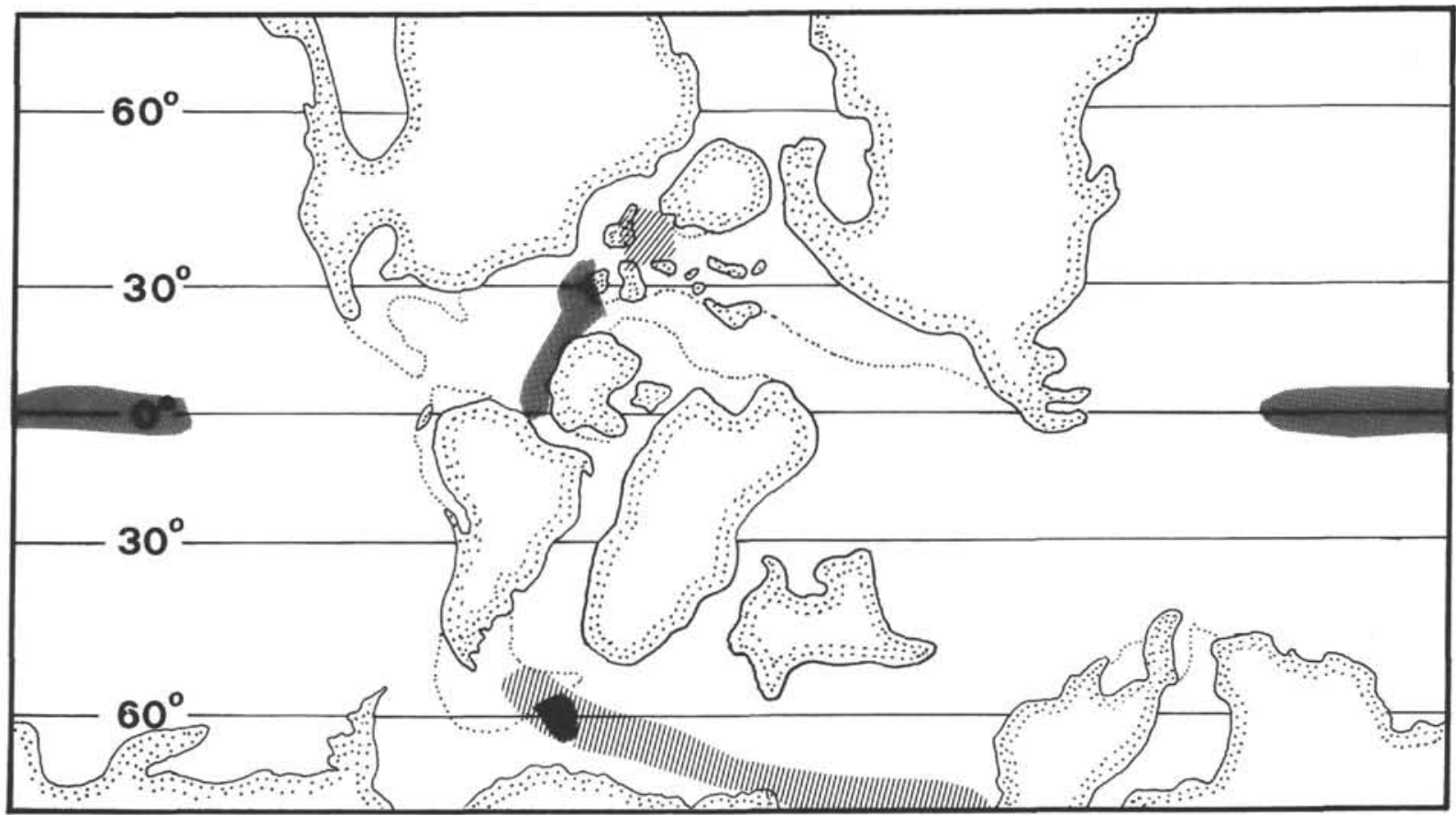

High-latitude assemblages in the middle Cretaceous.

High-fertility assemblages (upwelling and divergences) in the middle Cretaceous. High-fertility nannofossil indices: Biscutum constans and Zygodiscus erectus.

Figure 4. Synthesis of calcareous nannofossil biogeography for the middle Cretaceous (slightly modified after Roth, 1989). 


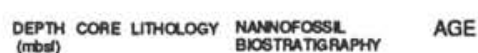

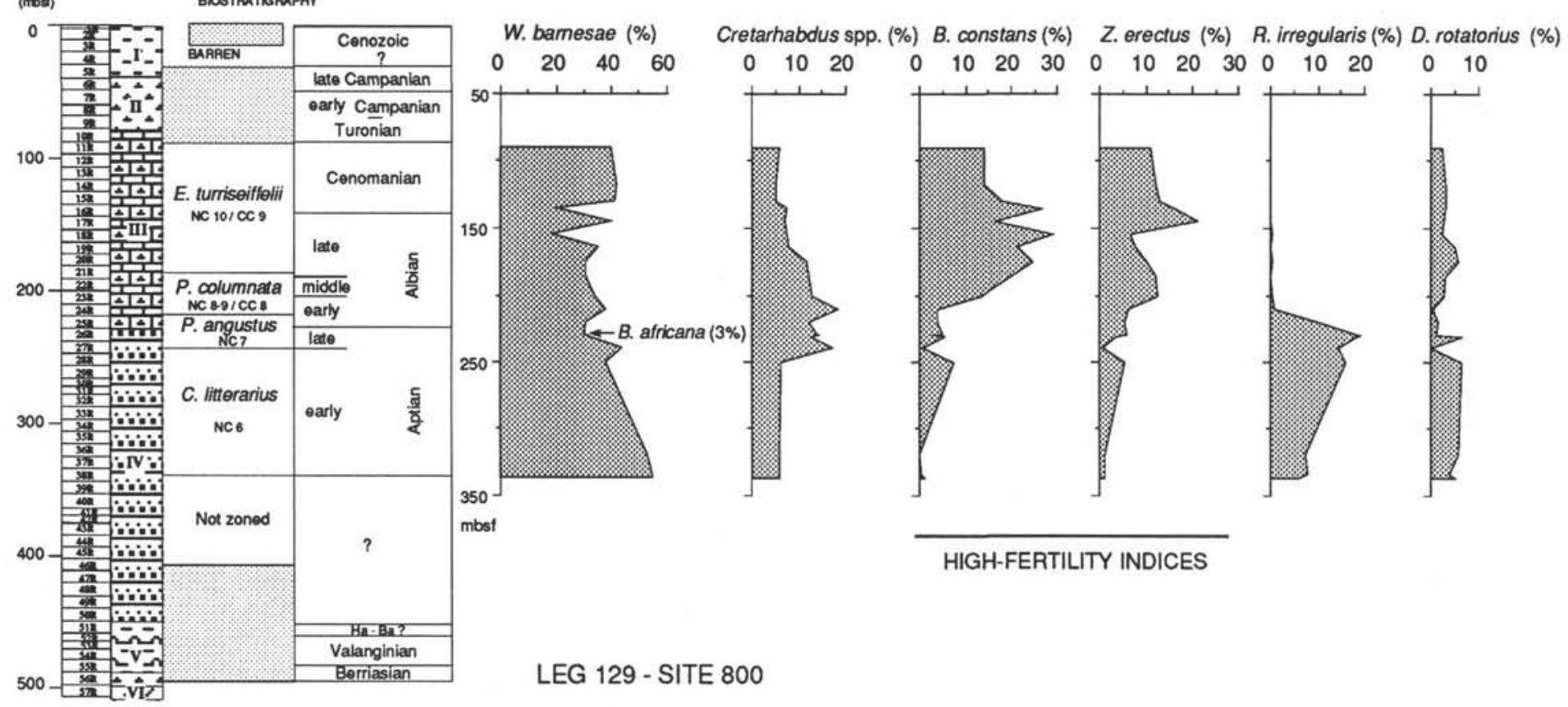

Figure 5. Distribution of the most abundant nannofossil taxa counted in the Aptian to Cenomanian interval at Site 800 . 


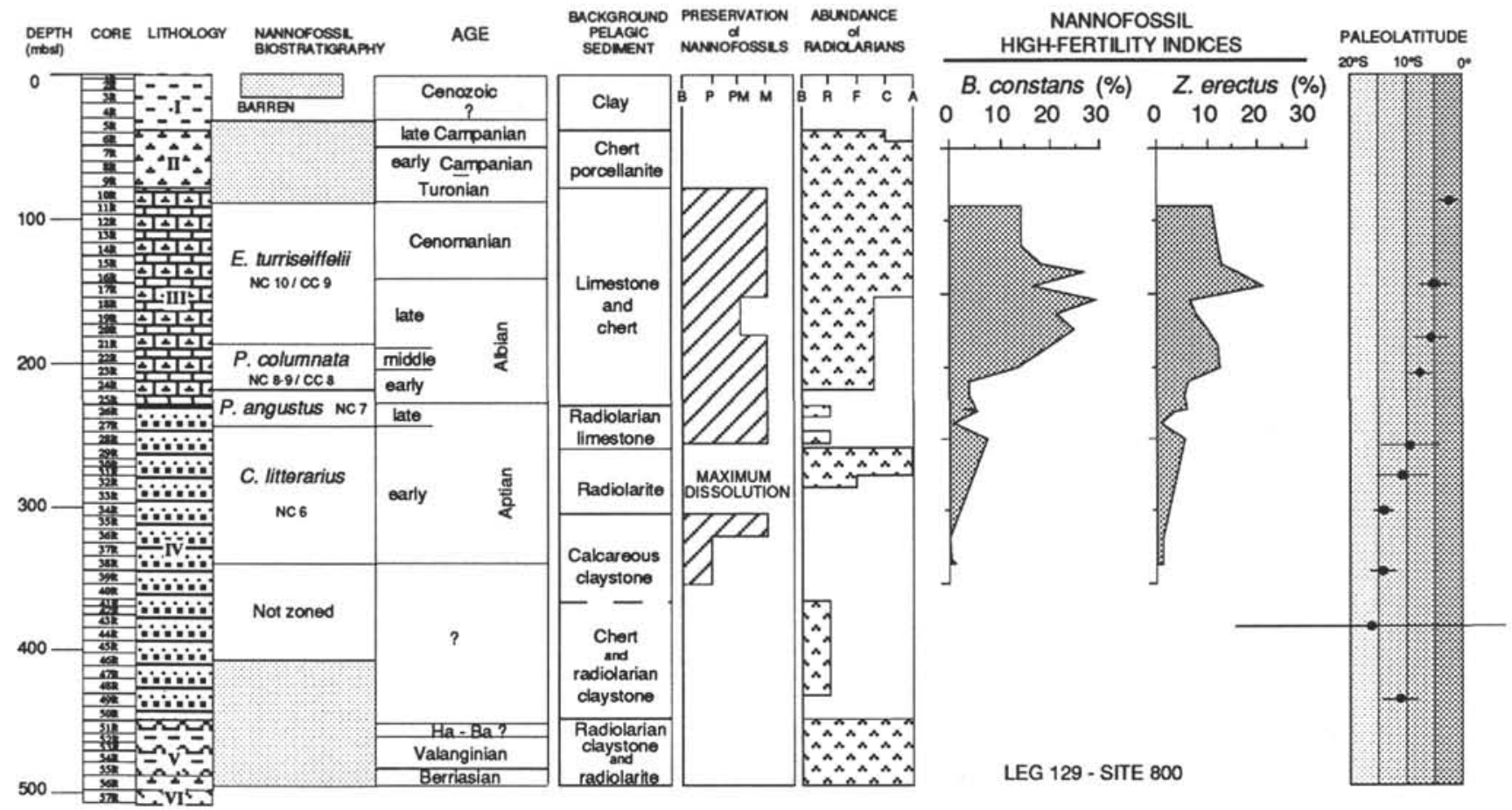

Figure 6. Changes in preservation of nannofloras and abundance of fertility indices in the Aptian to Cenomanian interval at Site 800. Distribution of radiolarians ("Biostratigraphy" section, "Site 800" chapter, Lancelot, Larson, et al., 1990) and paleolatitudes calculated from sediment inclinations ("Paleomagnetics" section, "Site 800" chapter, Lancelot, Larson, et al., 1990) are also shown.

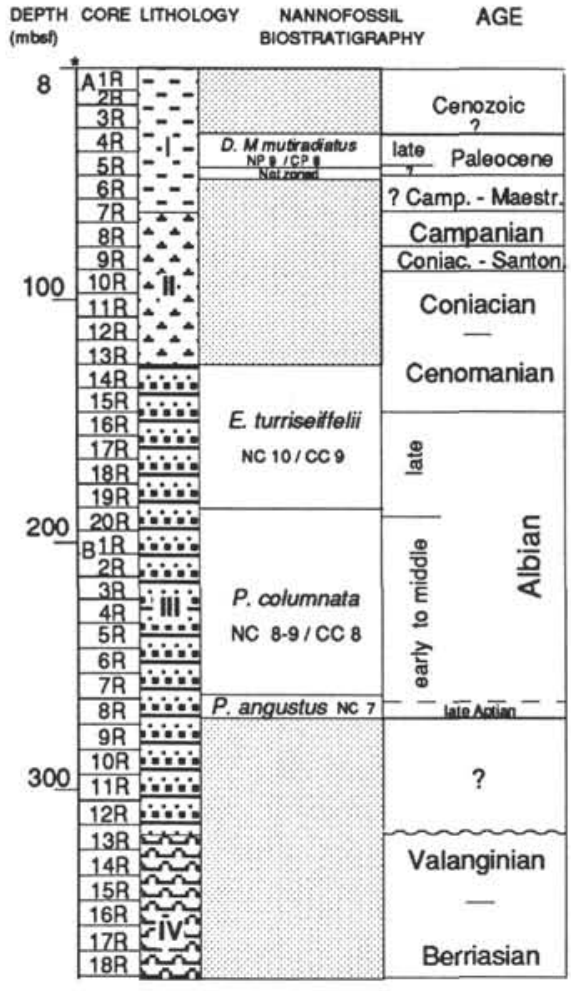

- No recovery between 0 and 8 mbst.
LEG 129 - SITE 801

W. barnesae (\%) $\quad$ B. constans (\%) Z. erectus (\%) Cretarhabdus spp. (\%) D. rotatorius (\%)

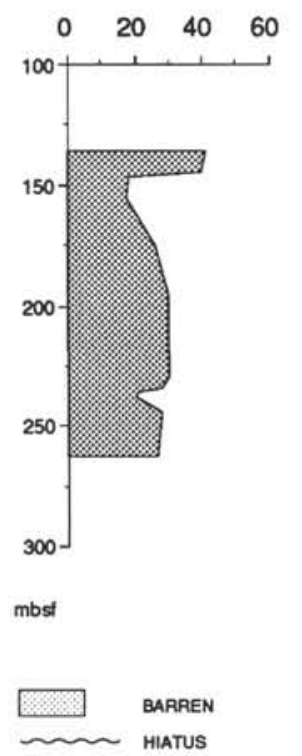

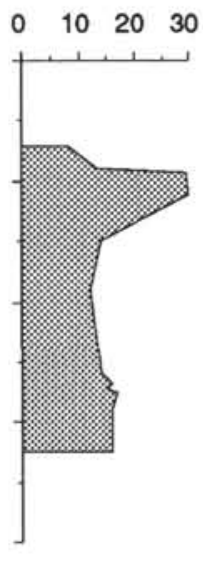

$0 \quad 1020$

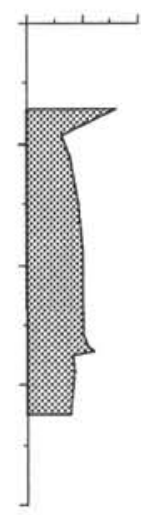

HIGH-FERTILITY INDICES

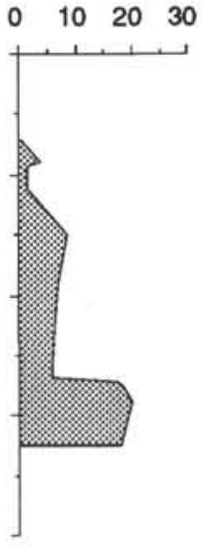

Figure 7. Distribution of the most abundant nannofossil taxa counted in the uppermost Aptian to Cenomanian interval at Site 801. 


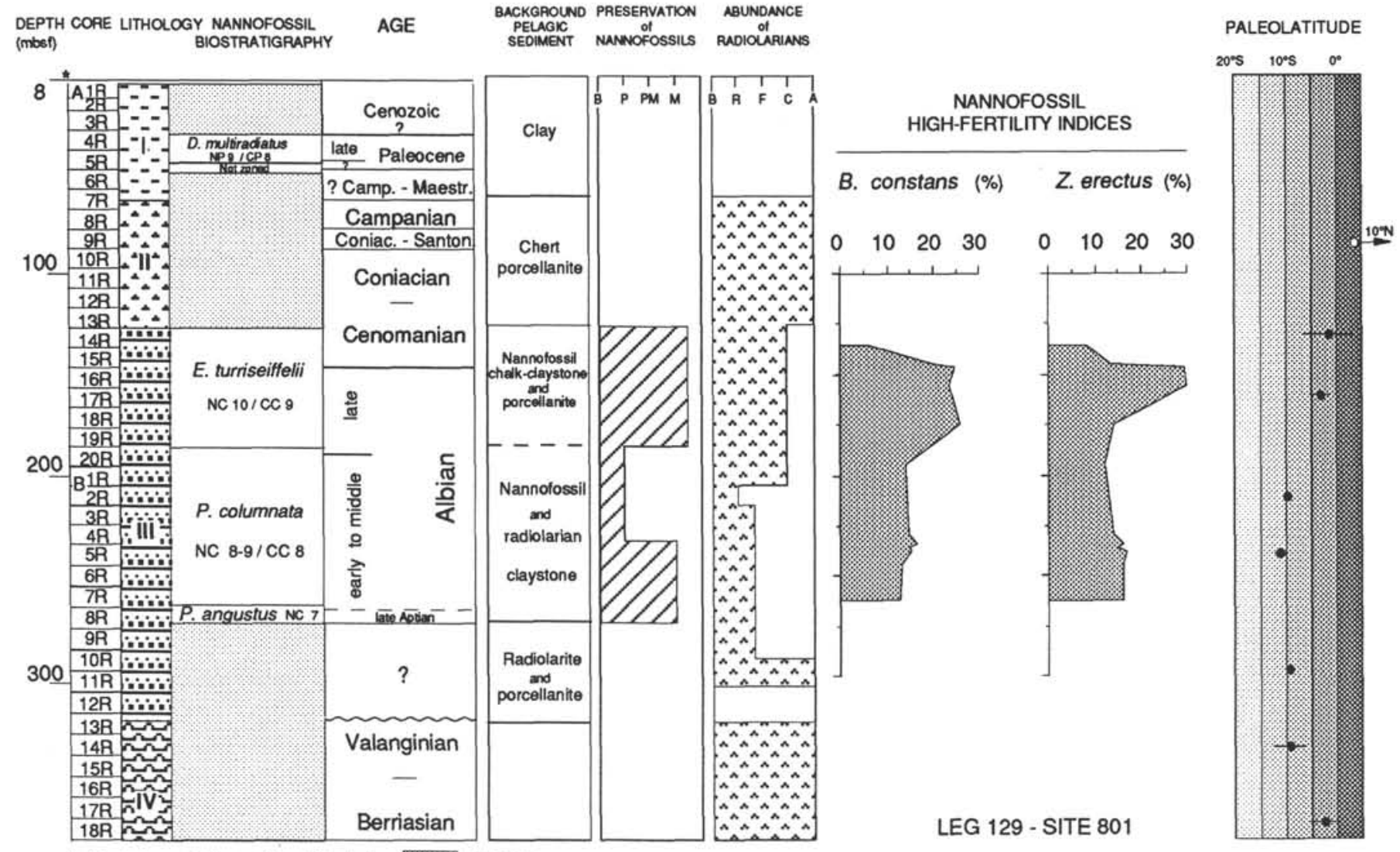

- No recovery between 0 and 8 mbsf. $\square$ BARREN

Figure 8. Changes in preservation of nannofloras and abundance of nannofossil fertility indices in the uppermost Aptian to Cenomanian interval at Site 801. Distribution of radiolarians and paleolatitudes are plotted after Lancelot, Larson, et al. (1990; "Biostratigraphy" and "Paleomagnetism" sections, "Site 801" chapter).

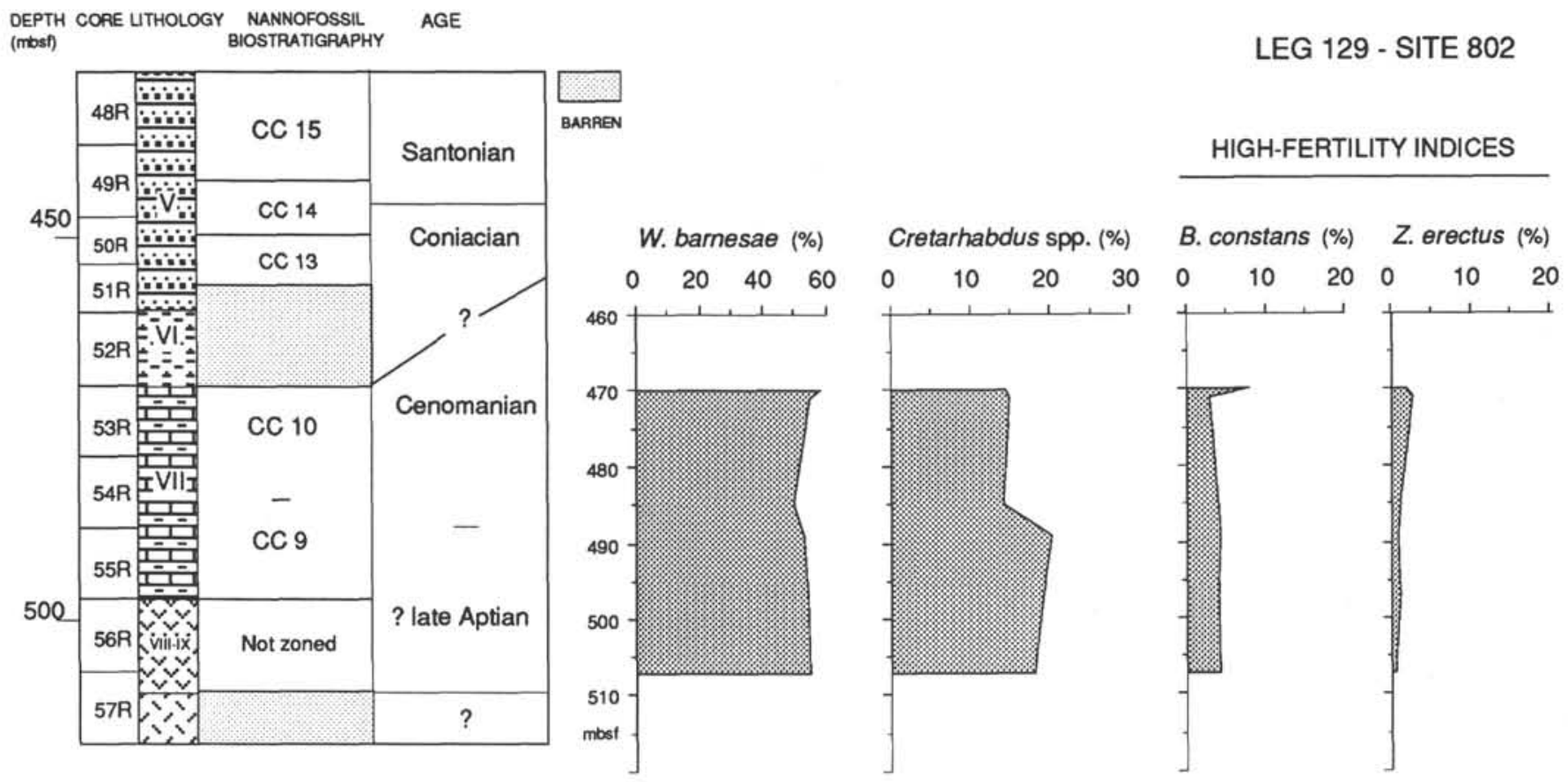

Figure 9. Distribution of the most abundant nannofossil taxa counted in the uppermost Aptian to Cenomanian interval at Site 802. 


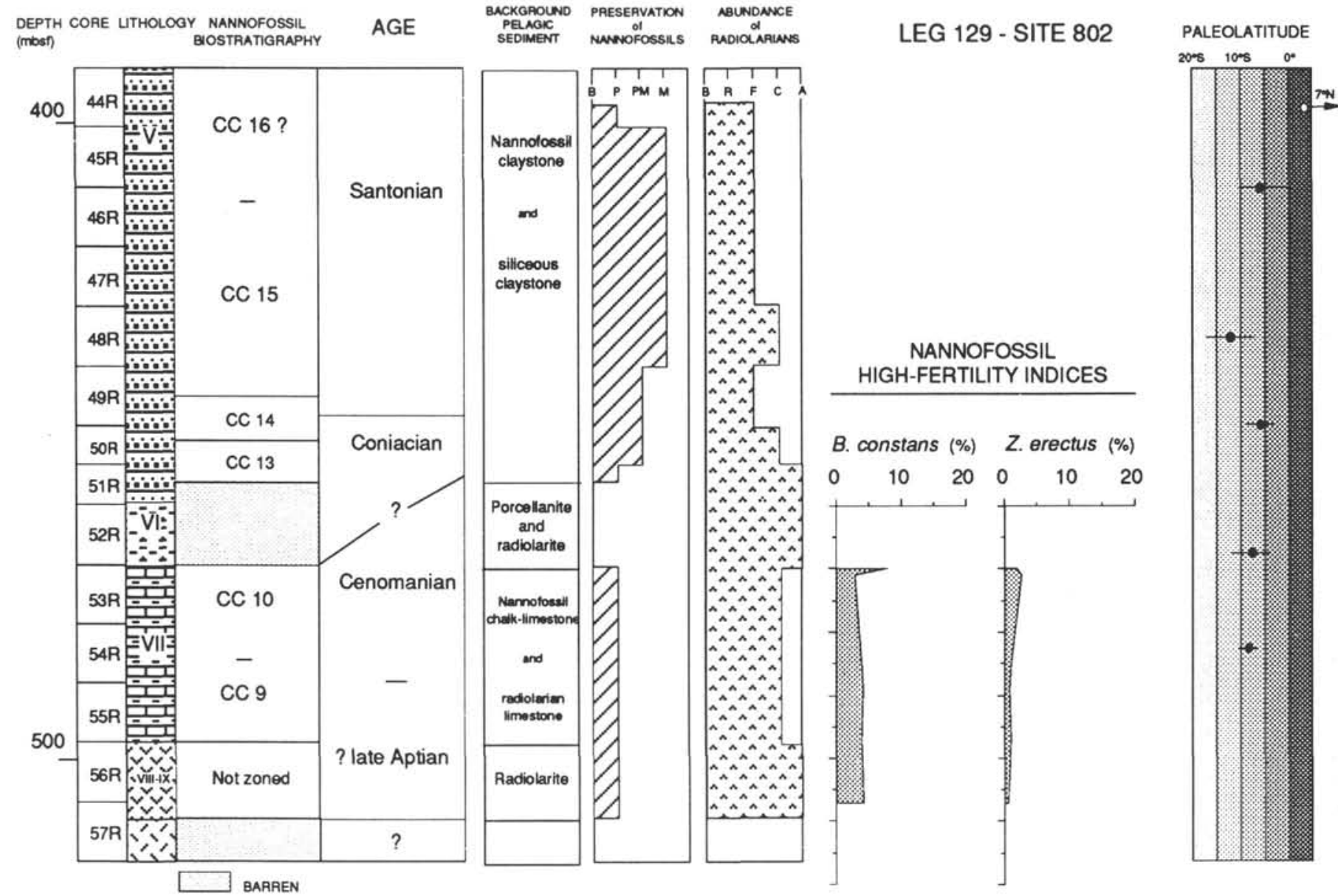

Figure 10. Changes in preservation of nannofloras and abundance of fertility indices in the uppermost Aptian to Cenomanian interval at Site 802. Distribution of radiolarians and paleolatitudes are plotted after Lancelot, Larson, et al. (1990; "Biostratigraphy" and "Paleomagnetism" sections, "Site 802" chapter). 


\section{TROPHIC RESOURCE CONTINUUM}

\begin{tabular}{|c|c|c|c|c|c|c|}
\hline CHLOROPHYL & 0.001 & 01 & & & & 100 \\
\hline $\begin{array}{l}\text { CONCENTRATIONS } \\
\left(\mathrm{mg} / \mathrm{m}^{3}\right)\end{array}$ & HIGHLY & OLIGOTROPHIC & MESOTROPHIC & EUTROPHIC & HIGHLY & \\
\hline & OLIGOTROPHIC & & & & EUTROPHIC & \\
\hline & & & REDUCED MIXI & & & \\
\hline & & & STRO & MIXING & & \\
\hline & & & MODERN PAC & & & \\
\hline RECENT & & & MODERN ATLA & & & A \\
\hline
\end{tabular}

\section{CRETACEOUS}

FORAMINIFERS

PLANKTONIC

globigerinelloids hedbergellids

NANNOFOSSILS

B. constans - Ex

$Z$. erectus

SILICEOUS ORGANISMS

RADIOLARIANS + DIATOMS

Figure 11. A. Representation of the oceanic surface-water trophic-resource continuum (TRC), comparing the modern Atlantic and Pacific oceans, and predicting how reduced oceanic mixing should expand the TRC while increased rates of oceanic mixing should reduce the TRC (adapted from Hallock, 1987). B. Inferred life-history strategies and trophic resources for the mid-Cretaceous. In the planktonic foraminiferal assemblages the hedbergellids seem to be adapted to resource-richer, lower stability environments with eutrophic niches, while the globigerinelloids seem apparently adapted to more mesotrophic conditions than hedbergellids (Caron and Homewood, 1983; Leckie, 1987, 1989; Premoli Silva et al., 1989; Sliter and Premoli Silva, 1990). In calcareous nannofossil assemblages, Biscutum constans and Zygodiscus erectus seem to prefer eutrophic conditions, partially overlapping with radiolarians' preferred environment. Moreover, $Z$. erectus seem to indicate more eutrophic conditions with respect to $B$. constans. 